\title{
Editorial
}

\section{Clinical Factor 2013}

\author{
Richard Balon \\ Departments of Psychiatry and Behavioral Neurosciences, and Anesthesiology, Wayne State University School of \\ Medicine, Detroit, Mich., USA
}

Continuing in what could be called almost a tradition after I have presented this treatise on the 'clinical factor' three times [1-3], I am presenting my subjective selection this time of 20 (primary) articles published in the previous year (this time in 2013) that I believe may provide some information to help the reader improve his/her clinical practice. As I noted before, this was originally proposed by Giovanni Fava [4] with the goal of selecting papers that I would subjectively perceive as having a high clinical factor - the degree and extent to which a journal article provides information to the clinician that may improve his/her practice. As I wrote before and as applies again [1]: 'Many may find them useful, some will find them less useful or useless. I am certainly biased, as is anybody else. I also cannot review all psychiatric journals - I focused only on most of the major ones. If there is no article selected from a certain major psychiatric journal, it does not necessarily mean that I have not reviewed it or skimmed through that journal. It may mean that I did not find, in my opinion, anything clinically very interesting.' This year I again expanded the number of journals I reviewed for clinically interesting and relevant articles. The journals that may or may not provide an article for this selection include the following: Acta Psychiatrica Scandinavica, American Journal of Psychiatry, JAMA Psychiatry (formerly Archives of General Psychiatry), British Journal of Psychiatry, Depression and Anxiety, European Psychia-

\section{KARGER}

E-Mail karger@karger.com

www.karger.com/pps try, General Hospital Psychiatry, JAMA, Journal of Affective Disorders, Journal of Clinical Psychiatry, Journal of Clinical Psychopharmacology, Journal of Psychosomatic Research, Journal of Nervous and Mental Disease, Journal of Sex and Marital Therapy, Psychological Medicine, Psychosomatics and Psychotherapy and Psychosomatics. I view myself first and foremost as a clinician (though academically oriented), and I am, as most clinicians are, looking for a way to help patients and for guidance in the treatment of my patients, and thus seeking new, innovative, or newly validated treatments. Thus, this is part of my clinician's bias and it impacts my selection: the majority of the selected articles are on treatment-related issues or long-term outcome. I had originally selected more articles, and it has been difficult to narrow them down to the final 20. I am sure I missed some interesting ones, but as noted, this is a subjective selection.

\section{Anorexia Nervosa}

The study by Franko et al. [5] from the Massachusetts General Hospital reminds us that anorexia is a deadly disease. In their long-term study (with a median follow-up of 20 years) of 246 treatment-seeking females with anorexia nervosa or bulimia nervosa, 16 deaths $(6.5 \%)$ were recorded - 14 in the group of 186 lifetime anorexia nervosa wom- (c) 2014 S. Karger AG, Basel

0033-3190/14/0836-0330\$39.50/0
Richard Balon, MD

Departments of Psychiatry and Behavioral Neurosciences, and Anesthesiology Tolan Park Building, Third Floor, 3901 Chrysler Service Drive Detroit, MI 48201 (USA)

E-Mail rbalon@wayne.edu 
en and 2 in the group of 60 bulimia nervosa women. This actually translates into $7.5 \%$ of the women with anorexia nervosa and $3.3 \%$ of the women with bulimia nervosa. Individuals with anorexia nervosa had significantly elevated mortality (6.2 times the expected rate), while those with bulimia nervosa did not ( 1.5 times the expected rate). Four of the deaths in the anorexia group were by suicide! The risk of premature mortality in anorexia appeared to decrease over time. As the authors noted, though, 'all deaths occurred in middle adulthood, with all but three deaths occurring between ages 35 and 48 years, suggesting that for women with long-standing histories of eating disorders, middle adulthood is particularly high-risk period for dying' (p. 924). The causes of death may have been mostly related to eating disorder symptoms. Multivariate predictors of mortality included alcohol abuse, low BMI, and poor social adjustment. [This study was supported by two National Institute of Mental Health (NIMH) grants.] As Crow [6] noted in the accompanying editorial, the risk of suicide in eating disorders may not be as well appreciated as in other psychiatric illnesses and thus deserves greater attention. The results also suggested that suicide mortality in eating disorders may be concentrated early in the illness, and thus intensive monitoring for suicidality early in the illness may be critical. Finally, he advocated the use of psychotherapies (especially family therapy) in adolescence as opposed to adulthood in this population. According to Crow [6], 'there is evidence that such therapies could be more effective than corresponding therapies delivered to adults; the possibility of diminishing overall mortality by intervening early lends further emphasis to the importance of such efforts' (p. 825). (Crow receives research support from Alkermes, Novartis, and Shire, though there was no evidence of influence from these relationships.)

Addressing the need for a specific psychotherapy for anorexia nervosa, Dalle Grave et al. [7] compared two inpatient cognitive behavior therapies (CBT) for anorexia nervosa: a focused form (CBT-Ef) exclusively targeting eating disorder psychopathology and a more complex broad form (CBT-EB) that also addresses some additional problems (e.g., mood intolerance, perfectionism, low self-esteem, and interpersonal difficulties) which in some subjects appear to maintain the eating disorder psychopathology. They randomized 80 patients ( 78 women) to these two modalities; most of the patients completed 20 weeks of treatment, and of those, most completed 6 and 12 months of follow-up after discharge. Psychotropic medications were not prescribed during the active treatment. Both therapies were acceptable to the patients, and they responded well to them: their mean weight gain was $12.7 \mathrm{~kg}$ (the initial mean weight for the whole sample was $37.4 \mathrm{~kg}$ ), and $80 \%$ of the patients had minimal eating disorder psychopathology at discharge. However, the follow-up results were a bit disappointing: the improvements in eating disorder psychopathology were sustained over the follow-up period, but the body weigh fell somewhat (by a mean of $3.3 \mathrm{~kg}$ for the entire group) during the first 6 months, though it stabilized in the whole sample at that point. Finally, there was no difference between the two versions of CBT at any point. While there are numerous limitations of this study (e.g., no comparison group treated with other modalities and the short follow-up period), the inpatient CBT seemed to be a helpful modality for patients with severe anorexia nervosa. (Two authors of this study were supported by the Wellcome Trust, but this foundation had no role in the design or execution of the study.)

In a multisite (Sydney, Australia, and London, UK) randomized controlled trial, Touyz et al. [8] compared CBT and specialist supportive clinical management in 63 female patients with severe and enduring anorexia nervosa (at least a 7-year illness history). All patients met the DSM-IV criteria for anorexia nervosa, excluding criterion D (amenorrhea) - this actually conforms to the DSM5 diagnosis, which does not require the presence of amenorrhea. The patients were seen during 30 outpatient visits spread over 8 months and at 6 - and 12-month posttreatment follow-ups. The major outcome measures were quality of life, mood symptoms, and social adjustment, while weight, eating disorder psychopathology, and other measures were considered secondary outcomes. Less than half of the patients were on psychotropic medications. There were no between-group differences at the end of the 8-month treatment. The CBT patients had higher scores on a social adjustment scale at the 6-month follow-up and lower Eating Disorder Examination scores and a higher readiness for recovery at the 12-month follow-up than the patients in the specialist supportive clinical management group. Interestingly, while the BMI improved moderately during the treatment, it remained basically unchanged during both follow-ups in both groups. While the CBT was superior in reducing core eating disorder symptoms at follow-up, both modalities helped over time in health-related quality of life, body weight, depression, and motivation to change. (Funding for this study was provided by the Australian National Health and Research Council, South West London, and St. George's NHS Trust, the Butterfly Foundation, and the University of West Sydney.) 
Both studies by Dalle Grave et al. [7] and Touyz et al. [8] suggest that specialized psychotherapy, especially CBT, could be helpful in the management of severe anorexia nervosa.

\section{Depression, Pregnancy, Medications, and Risks to Infants}

The impact of maternal depression and of medication on children has been a hotly debated issue for a long time. Pearson et al. [9] studied the risks and possible mechanisms of offspring depression at the age of 18 years in children of mothers with depression during pregnancy and the postnatal period. They asked four questions: (1) are maternal antenatal depression (AND) and maternal postnatal depression (PND) associated with offspring depression at the age of 18 years; (2) do AND and PND have independent effects on offspring depression, and if so, are the risks of a different magnitude; (3) does maternal education moderate the effects of PND but not AND, and (4) are the effects of AND, but not PND, unique to mothers? They used data from a large community-based birth cohort: the starting sample of those with maternal AND and PND included 8,937 individuals; outcome data were available for 4,566 adolescents, complete data across all exposure, outcome, and confounding variables were available for 2,847 individuals, and data on paternal depression and education were available from 2,475 individuals. Depression in mothers was evaluated using the Edinburgh Postnatal Depression Scale, and depression in the offspring was assessed using the Clinical Interview Schedule-Revised. The results showed a main effect of both AND and PND scores on offspring depression, and both associations were independent of later depression. The intercepts of mothers' depression trajectories from the antenatal to the postnatal period were positively associated with the risk of offspring depression at the age of 18 years. As a moderating effect, there was evidence that the effect of PND was limited to mothers with lower education (the effects of AND were present in mothers with both lower and higher education). There was no association between paternal AND and offspring depression; yet there was evidence for an association between paternal PND and offspring depression, though only for offspring of fathers with low education. The authors pointed out that their study did not test the mechanism of the transmission of depression from mother to adolescent. Based on the fact that education moderated the effects of PND, the authors felt that the effect of environmental factors was strong. It seems that AND is an independent risk factor for offspring depression, while PND may be a risk factor only in disadvantaged families. The authors suggested that treating depression in pregnancy may be the most effective intervention to prevent depression in offspring. As the association between PND and offspring depression appears be greatest for children of mothers with lower education, the treatment may be prioritized to more disadvantaged mothers. However, the preventive effect of the treatment of depression in pregnancy remains to be tested. (This study was supported by grants from the Wellcome Trust, from the National Institutes of Health, and from the UK Medical Research Council.)

Though the treatment of depression during pregnancy seems advisable, the important question is whether (and if so, how) the treatment itself is harmful to the offspring. Selective serotonin reuptake inhibitor (SSRI) treatment of pregnant women has been associated with adverse outcomes in infants (e.g., neonatal withdrawal syndrome, pulmonary hypertension in the newborn with some SSRIs, a higher risk of preterm birth, and higher rates of delivering low-birth-weight infants). On the other hand, discontinuation of antidepressant treatment increases the risk of relapse of depression in pregnant women. Maternal depression has also been associated with slower rates of fetal body and head growth and with an increased risk of preterm delivery, which may cause neonatal morbidity and mortality. Stephansson et al. [10], in their population-based cohort study in all Nordic countries (Denmark, Finland, Iceland, Norway, and Sweden; these countries have similar nationwide registries of births and dispensed drugs), investigated the risk of stillbirth and infant mortality associated with the use of SSRIs during pregnancy. They focused on singletons born after 154 gestational days in different time periods in different countries between 1996 and 2007. A mother's exposure to SSRIs was defined as 1 or more filled prescriptions for an SSRI from 3 months before the start of pregnancy until birth. Maternal data also included possible confounders such as maternal age, smoking, and parity. The authors obtained data on $1,633,877$ singleton births: 6,054 were stillbirths, 3,609 neonatal deaths, and 1,578 postneonatal deaths. A total of $29,228(1.79 \%)$ of the mothers filled out a prescription for an SSRI during pregnancy. Women exposed to an SSRI had higher rates of stillbirth (4.62 vs. 3.69 per $1,000 ; \mathrm{p}=0.01)$ and of postneonatal deaths (1.38 vs. 0.96 per 1,$000 ; \mathrm{p}=0.03)$ than those who were not. The rate of neonatal deaths was similar. However, in a multivariate analysis, SSRI use was not associated with stillbirth, neonatal death, or postneonatal death. The increased rates of 
stillbirths and postneonatal deaths were explained by the severity of the underlying maternal psychiatric disease and other risk factors such as cigarette smoking and advanced maternal age. While this study did not include data on spontaneous abortion - and information on dispensed medications is not necessarily the same as information on their intake - it still presents strong evidence that exposure to SSRIs during pregnancy is not associated with an increased risk of stillbirth and infant mortality. (This study was funded by the Swedish Pharmacy Company and by the authors' affiliated institutions. The lead author was supported by a postdoctoral fellowship from the Swedish Society of Medicine. The Swedish Pharmacy Company was not involved in the design of the study, analysis of the data, or writing of the manuscript.)

In another study, this time a prospective observational one, Wisner et al. [11] compared the growth of infants born to women with antenatal major depression, either treated or untreated with SSRIs, and infants born to nondepressed, nonmedicated women. They evaluated 97 nondepressed, nonmedicated women, 46 women treated with SSRIs, and 31 depressed women without exposure to SSRIs at weeks 20,30, and 36 of gestation, and then mother-and-infant pairs were assessed at 2, 12, 26, and 52 weeks postpartum (they included only women with sufficient longitudinal data). The growth assessment included infant weight, length, and head circumference. There were no differences in growth assessment between the three groups; thus, no significant association between prenatal maternal SSRI exposure or depression and the growth variables (weight, length, or head circumference) from birth through 12 months of age was observed. While the results are interesting and important, there are a few caveats/limitations. The sample was fairly small. It is also not clear to which specific SSRIs the observed women were exposed and whether there was any effort to avoid some SSRIs such as paroxetine known to be associated with a serious complication (pulmonary hypertension). (This study was supported by an NIMH grant. Some authors reported conflicts of interest for various entities, all unrelated to this study.) As Parry [12] noted, the findings by Wisner et al. [11] suggest that factors regulating infant growth are robust enough not to be significantly perturbed by major depression or its treatment with SSRIs. However, Parry [12] added (citing Stephansson et al. [10]) that any decision about using SSRIs during pregnancy must take into account other perinatal outcomes and the risk associated with maternal mental illness.

Another population-based study from Denmark [13] provides important and interesting information about prenatal exposure to valproate. Valproate is associated with an increased risk of congenital malformations following fetal exposure. Most psychiatrists avoid prescribing valproate during pregnancy for this reason, and it is classified as Pregnancy Category D in the USA if used for the treatment of epilepsy or bipolar disorder. Christensen et al. [13], in their study of 655,615 children born between 1996 and 2006, identified 5,437 children with autism, including 2,067 with childhood autism (the mean age at the end of the follow-up was 8.8 years; range: $4-14$ ). The estimated absolute risk of autism after 14 years of follow-up was $1.53 \%$ for autism spectrum disorder and $0.48 \%$ for childhood autism. However, if analyzing the data for 508 children exposed to valproate, the absolute risk for autism spectrum disorder was $4.42 \%$ and $2.5 \%$ for childhood autism. In the cohort of 6,584 children born to women with epilepsy, the absolute risk of autism spectrum was $4.15 \%$ and the risk of childhood autism was $2.95 \%$ in 432 children exposed to valproate, while the absolute risk of autism spectrum disorder was $2.44 \%$ and that of childhood autism $1.02 \%$ in 6,152 children not exposed to valproate. Thus, maternal use of valproate during pregnancy was clearly associated with a significantly increased risk of autism spectrum disorder and childhood autism, even after adjusting for maternal epilepsy. Even after adjusting for parental psychiatric history (a well-known risk factor for autism in offspring), the estimates of the risk associated with valproate exposure changed little. (This study was supported by grants from the European Research Council and the Danish Medical Research Council. Christensen received support from the Danish Epilepsy Association, and Pedersen from the Danish Council for Independent Research.) The limitations of this study, pointed out by Meador and Loring [14], include inadequate information about the periconceptional use of folate, the use of alcohol or illicit drugs during pregnancy, and possible missed psychiatric diagnoses in the parents. Nevertheless, the result expands our data about the dangers of prescribing valproate during pregnancy. As Meador and Loring [14] write, 'because approximately half of the pregnancies in the United States are unplanned, delaying discussions of treatments risks until a pregnancy is considered will leave a substantial number of children at unnecessary risk. Women of childbearing potential should be informed of the potential risk of fetal valproate exposure before valproate is prescribed.' (While Meador and Loring received support from the NIH/NINDS as well as various pharmaceutical companies and institutions, none seems to be related to the writing of their editorial.) 


\section{Suicide}

Lately, the ongoing debate on the possible prevention of suicide has focused (especially in the USA) on the availability of firearms. At times, legislative and other societal decisions may have unintended consequences and provide data even about issues such as suicide prevention. One such example is described in a report by Reisch et al. [15] from Switzerland. Guns are widely available in Swiss homes as militia soldiers (used for country defense) store their guns at home. Approximately every other household in Switzerland owns a gun (a total of approx. 2 million guns). In 2003, the Swiss army was restructured, and its troops were reduced from approximately 400,000 to 200,000 . This reduction had a significant impact on the availability of military guns. In addition, the discharge age was lowered from the age of 43 to the age of 33 years, the fee to purchase guns by soldiers upon discharge was increased, and gun licenses are required. Following this decrease in the availability of guns, Reisch et al. [15] compared the suicide rates before (1995-2003) and after the intervention (2004-2008) in the affected population of men aged 18-43 years and in two comparison groups: women aged 18-44 years and men aged $44-53$ years. They found reductions in both the overall suicide rate and the firearm suicide rate: the overall suicide rate decreased by 2.16 per 100,000 and the suicide by firearm rate decreased by 2.64 per 100,000 (from 9.9 to 7.26 per 100,000, a $27 \%$ decrease). There was a slight increase in suicide by other means $(0.48 \%)$. Importantly, approximately 30 men in the age group from 18 to 43 years each year did not die by suicide but might have otherwise. There were no significant differences in overall and by-firearm suicide rates in the female control group. The comparison group of men aged 44-53 years had a marginal decrease in the rate of suicide by firearm (1.43, not significant after Bonferroni correction), but a significant increase of suicide by other methods (namely, railway suicides). This group's overall rate increase was not significant after Bonferroni correction. The authors noted that since the intervention' was an unintended consequence of the Army XXI reform, the possibility of a decrease in suicide rate due to causes such as greater awareness of the suicide problem could be excluded. (This study was supported by a grant from the Swiss National Science Foundation.) In an accompanying editorial, Mann and Gibbons [16] note that if the results of this study were to be applied to the USA, where the 2010 firearm suicide rate for the same group was 11.06 per 100,000 , the rate would decrease to 8.07 per 100,000 , saving 1,636 lives per year on average!
They also point out that people living in households with a firearm have almost 5 times the risk of suicide than people living in gun-free homes, and that for every case of a firearm being used in self-defense homicide, there were 37 firearm suicides. Last but not least, they noted that given the high rates of suicide in US veterans and the growing number of veterans, the problem of firearm suicide deserves much more attention. (Gibbons has served as an expert witness for the US Department of Justice, Pfizer, and Wyeth on cases related to suicide. Mann has received research support from GlaxoSmithKline and Novartis and receives royalties for the Columbia Suicide Severity Rating Scale from the Foundation for Mental Health.)

The Saving and Empowering Young Lives in Europe (SEYLE) study is a randomized clinical trial to assess prevention strategies for suicidal behavior in 11 countries. Selected school classes (regardless of size) in which at least $50 \%$ of the pupils were aged 14 years were surveyed using various tools such as the Paykel Suicide Scale and the Strengths and Difficulties Questionnaire. This age group was selected because of its risk propensity for suicidal behavior. Kelleher et al. [17] report results from the Irish site, as questions on psychotic symptoms (Adolescent Psychotic-Like Symptom Screener) were included only for the Irish center. Pupils in 17 randomly selected schools in Counties Cork and Kerry were tested at the baseline and 3- and 12-month follow-ups. The researchers distributed 1,602 consent forms, and 1,112 students took part in the baseline evaluation (69\% response rate). Of these, $90 \%(1,006)$ completed the 3-month follow-up and $88 \%$ (973) completed the 12 -month follow-up. Of the total sample of 1,112 students, $7 \%$ reported psychotic symptoms at baseline. Of that subsample, $7 \%$ reported a suicide attempt by the 3 -month follow-up compared with $1 \%$ of the rest of the sample, and $20 \%$ reported a suicide attempt by the 12 -month follow-up compared with $2.5 \%$ of the rest of the sample. Of the participants with psychopathology at baseline who did not report psychotic symptoms, 3.5\% reported a suicide attempt by the 3-month follow-up and 135 by the 12-month follow-up, while of those with psychopathology at baseline who reported psychotic symptoms, $14 \%$ reported a suicide attempt by the 3-month follow-up and $34 \%$ reported a suicide attempt by the 12-month follow-up! The result for the 12 -month follow-up compares with $4 \%$ of the total general population and $13 \%$ of the participants with psychopathology who did not report psychotic symptoms at baseline. Adolescent with psychopathology who reported psychotic symptoms had nearly 70 -fold increased odds of 
acute suicide attempts. In spite of various limitations (self-report, sample limited to one geographic location, and culture), these are striking numbers. As the authors suggest, adolescents with psychotic symptoms are at high clinical risk of suicide attempts, and thus more careful clinical assessments of psychotic symptoms, attenuated or frank, and of suicidal ideation and plans are required. (This study is supported through the Coordination Theme of the EU Seventh Framework Programme.)

In an interesting article from Britain, Roberts et al. [18] report on changes in high-risk occupations for suicide over the past 30 years. In the past (during 1979-1980 and 1982-1983), several occupations with easy access to a method of suicide (pharmaceuticals, guns) had the highest rates of suicide in Britain. These included veterinarians (77 per 100,000 worker-years), followed by merchant seafarers (76), hotel porters (74), pharmacists (46), hospital porters (37), dentists (36), roofers (32), and doctors (31). During the 2001-2005 period, the highest rates of suicide were among coal miners ( 81 per 100,000 workersyears), followed by other manual occupations such as merchant seafarers (68), laborers in building trades (59), and window cleaners (46). Interestingly, of the 'high-risk' occupations ( $>20$ per 100,000 in either of the two study periods), several had a significant $(\mathrm{p}<0.05)$ reduction in suicide rates, e.g., electronic engineers (95\%), hotel porters $(89 \%)$, radiographers $(87 \%)$, veterinarians $(74 \%)$, doctors (63\%), dentists (59\%), and farmers (38\%). There were, however, also significant increases over time in suicide rates for 5 occupations: coal miners (224\%), laborers in building trades (200\%), plasterers (117\%), forklift drivers $(71 \%)$, and carpenters (34\%). All 5 occupations with significant increases in suicide risk were manual, while only 3 of the 13 occupations that had significant reductions were manual (statistically significant difference; Fisher's exact test; $\mathrm{p}=0.007$ ). The authors' explanations for these changes include various socioeconomic forces. Some industries have undergone serious changes, contraction, and reconfiguration, resulting in the loss of traditional patterns of employment. Other explanations for the increase in suicide rates in manual occupations include poor reemployment prospects as well as a reduced ability to change and manage stress. Interestingly, the increases in suicide rates occurred during a period of economic prosperity. However, wider social inequalities are known to be associated with poorer health and mental illness as well as increases in suicide. The interesting decrease in suicide rates in physicians and dentists awaits more exploration and explanation. (No funding for this study was acknowledged/needed.)

Clinical Factor 2013
It seems that some risks of suicide could be modified (the accessibility of guns and other means) or they change over time (occupation). Awareness and the screening of some risks (psychotic symptoms, gun availability, and occupation) should be included in prevention programs and clinical management.

\section{Cognition}

Medicine and psychiatry have been paying more attention to cognitive functioning lately, especially in the areas of schizophrenia (previously called 'dementia praecox') and, of course, dementias (or major cognitive disorders). However, cognitive impairment occurs in other psychiatric disorders (e.g., pseudodementia in major depression). Two small studies investigated cognitive functioning in major mood disorders. Hasselbach et al. [19] investigated whether the cumulative number, duration, and subtypes (severity and presence of psychotic features) of previous episodes of depression in patients with unipolar depressive disorder in a remitted state are associated with decreased global cognitive functioning. Through the Danish registry, they obtained a sample of 88 patients (first discharge from psychiatric hospital in one region between 1994 and 2002) and 50 gender- and age-matched controls. Data on the use of medications were obtained. Cognitive functioning was evaluated using the Cambridge Cognitive Examination [CAMCOG; the cognitive part of the Cambridge Mental Disorders of the Elderly Examination (CAMDEX)] and the Global Deterioration Scale (to compare cognitive functioning between participants and nonparticipants). In addition, the authors obtained the proxy estimates of the premorbid cognitive capacity of the participants by an estimate of educational level as measured on a 17-point scale and by the DART, the Danish version of the National Adult Reading Test, as a measure of premorbid IQ (it tests the correct pronunciation of a list of 50 irregular words). The cumulative duration of depressive episodes was associated with a decreased CAMCOG score adjusted for age, gender, education, premorbid IQ, and residual depressive symptoms. A significant association was also found between CAMCOG score and the cumulative duration and total number of episodes with psychotic features. Though the interpretation of the results is limited by the crosssectional design (no real baseline cognitive functioning assessment, just estimates), the results still strongly suggest progressive cognitive impairment during the course of recurrent depression, especially the one with psychotic 
features, independent of residual depressive symptomatology. (This study was funded by the Lundbeck Foundation, the Eli and Egon Larsen Foundation, the Aase and Einar Danielsen Foundation, and a University of Copenhagen SUND PhD Scholarship Grant.) In a more sophisticated design, Mora et al. [20] assessed cognitive functioning in 28 bipolar I or II disorder patients and 26 healthy matched controls at baseline and 6 years later using a cognitive battery tapping into main cognitive domains such as executive function, attention, processing speed, verbal memory, and visual memory [altogether 7 tests and 3 subtests of the Wechsler Adult Intelligence Scale (WAIS)-III]. All patients were euthymic [Hamilton Rating Scale for Depression (HAMD) score $<8$ and Young Mania Rating Scale score $<60$ for at least 3 months prior to both evaluations]. At baseline, 14 patients were on lithium monotherapy and 14 on a combination of lithium with antidepressants and/or antipsychotics. At the end of the study, 2 patients were drug free, 11 were on lithium monotherapy, 11 were on combination therapy of lithium with other medications, and 5 were receiving other treatment (mood stabilizers either alone or in combination with antipsychotics and/or antidepressants). Main effects were in the executive domain, in the inhibition domain, in the processing speed domain, and in the verbal memory domain $(\mathrm{p}<0.04)$. Only longer illness duration was significantly related to slow processing $(\mathrm{p}=0.01)$. There was a strong relationship between impaired cognition along time and poorer psychosocial functioning $(\mathrm{p}<$ 0.05 ). The authors speculated that the high unemployment rates of bipolar patients may reflect the impact of the disorder on psychosocial functioning and cognition (processing speed deficit, attention deficit). Limitations of the study include the small study population and lack of control for medication impact on cognition (though presumably small); on the other hand, the study was prospective and of a fairly long duration. [The study was supported by a Fundacio Marato de TV3 Grant, the IRBLleida (Biomedicine Research Institute) Contest for a research project for medical registers, and the Spanish FIC-MCS.]

Dregan and Gulliford [21] studied the association between different leisure-time physical activities (LTPA) from 11 to 50 years and cognitive functioning in late midadulthood using a prospective birth cohort study including participants in the UK National Child Development Study aged 11-50 years. They obtained data on 9,790 subjects (which could be considered an $80 \%$ response rate, as of the original sample of 17,638 children, 12,316 were still alive and traceable). Data about cognitive measures (cog- nitive, memory, and executive functioning) were collected using face-to-face interviewing with a trained researcher when the participants were 50 years of age. Data on LTPA were collected through face-to-face interviewadministered questionnaires at the ages of $11,16,33,42$, 46 , and 50 years. All measures were self-reported, except for the one at the age of 11 years, which was based on parental reports. There was a steady decline in the proportion of men and women who participated in LTPA for at least 4 days/week from childhood to the age of 46 years, followed by an increase in LTPA to the age of 50 years. After adjusting for confounding variables (e.g., social class, smoking, alcohol use, and BMI), multiple regression analyses showed a positive association between LTPA frequency and cognitive index, and also memory and executive functioning, for both men and women. The greatest benefit emerged from participating in lifelong intensive LTPA. As the authors noted, physical activity represents a key component of lifestyle intervention to prevent cognitive decline, cardiovascular disease, diabetes, and cancer, and thus its promotion is of paramount importance. (This study was supported by the National Institute for Health Research Biomedical Research Centre at Guy's and St. Thomas, the National Health Service Foundation Trust, and King's College London.)

One wonders whether LTPA could be helpful in preventing/remediating the cognitive decline in the mood disorder patients of the studies by Hasselbach et al. [19] and Mora et al. [20].

\section{Psychotherapy of Depression}

Several studies investigated the use of psychotherapies (mostly CBT) in major depression with particular focus on the prevention of relapse and recurrence. Driessen et al. [22] compared the efficacy of manualized individual CBT (164 patients) and short-term psychodynamic supportive therapy (177 patients) in adults with major depression (HAMD score $\geq 14$ ). Severely depressed patients (HAMD score $\geq 24$; 150 patients) were offered antidepressant medication, initially venlafaxine $(6.2 \%$ of them did not start it). Both psychotherapies were conducted in 1 of 3 outpatient clinics, randomly assigned (with stratification by gender and age), and comprised 16 individual sessions over 22 weeks. The primary outcome measure was posttreatment remission (HAMD score $\leq 7$ ), and secondary outcome measures included mean posttreatment HAMD and patient-rated depression scores and 1-year follow-up remission rates. About a quarter of the patients 
did not complete treatment (CBT: $31.1 \%$; psychodynamic psychotherapy: $25.9 \%$ ). Based on the observed data, $24.3 \%$ of the patients in the CBT group and $21.3 \%$ of the patients in the psychodynamic group met the remission criterion at the posttreatment assessment. Follow-up assessments were done on 192 participants (56.3\%). More patients in the CBT group (44.6\%) than in the psychodynamic group (33.0\%) reported receiving additional treatment during the follow-up period, but this difference was not statistically significant $(\mathrm{p}=0.10)$. Actually, no statistically significant differences were found for any of the outcome measures. There are several limitations such as the number of patients not completing treatment or being lost to assessment, the lack of blindedness of the HAMD assessors, possible selection bias, additional treatment during the follow-up sought by the patients, and the lack of a control group. While the study demonstrated some efficacy of both therapies, the average posttreatment remission rate of $22.7 \%$ was low, and a number of patients (40\%) sought additional treatment afterward. The authors attempted to explain the low remission rates by the low socioeconomic status and income levels in their sample. Nevertheless, these rates are disappointing. (This study was supported by an unrestricted grant from Wyeth Pharmaceuticals, The Netherlands, research grants from Arkin Mental Health Care, Amsterdam, a grant from Pro Persona Mental Health Care, and by grants from the Faculty of Psychology and Education, Department of Clinical Psychology, VU University Amsterdam.)

In another study of two psychotherapy modalities, Stangier et al. [23] compared the relapse and recurrence outcomes of maintenance manualized CBT (that included well-being therapy and mindfulness-based cognitive therapy) and manualized psychoeducation in addition to treatment as usual in a multicenter prospective randomized observer-blinded study with two parallel groups. Patients with 3 or more previous episodes of major depression who met the remission criterion over a 2-month baseline period were assigned to either maintenance CBT (87 started) or manualized psychoeducation (86 started). They received both therapies for 16 sessions (50 $\mathrm{min}$ for CBT, 20 min for psychoeducation) over 8 months. The therapies were conducted either by psychologists or psychiatrists. After completion, the patients were followed up for 12 months. The main outcome measure was time to first relapse or recurrence of major depression as assessed by blinded observers with the Longitudinal Interval Follow-Up Evaluation. Only 78 patients in the CBT group and 66 patients in the psychoeducation group completed at least 12 sessions, and only 74 patients in the
CBT group and 59 in the psychoeducation group completed the 1-year follow-up. The difference in 1-year dropout rates was significant $(\mathrm{p}<0.01)$. Three quarters of patients received antidepressants. There was no overall significant difference in time to relapse or recurrence of major depression between the groups (607 days from randomization for maintenance CBT and 531 days for manualized psychoeducation). However, maintenance CBT was significantly superior to manualized psychoeducation in a subsample of patients with 5 or more depressive episodes. One wonders how these subsamples were identified (post hoc?). One also wonders whether the difference in session length (50 vs. $20 \mathrm{~min}$ ) could have played any role in any of the differences. Notably, 2 patients died by suicide, 1 after discontinuing maintenance CBT and 1 after completing manualized psychoeducation. This should serve as a sober reminder that psychotherapies could also have adverse outcomes. Based on these results, it seems that maintenance CBT and manualized psychoeducation could be equally helpful in moderating the risk for relapse of depression, while CBT may be superior in patients with a higher risk of depression recurrence. Unfortunately, this study also did not include a treatmentas-usual comparison group, and the follow-up of 12 months was relatively short. (The study was supported by three German Research Funding grants.)

Jarrett et al. [24] reported that depressed patients in the continuation phase of cognitive therapy (C-CT; 86 patients) or on fluoxetine maintenance ( 86 patients) were both significantly less likely to relapse than patients on pill placebo (59 patients) over the 8 -month continuation treatment period (after acute-phase CT) and in the 24-month posttreatment follow-up. The patients selected for this study responded to the acute phase of CT (16-20 individual sessions over 12 weeks; each session: 50-60 $\mathrm{min}$ ) but were classified as at higher risk for relapse. They were thus randomly assigned to the three treatment arms at two different clinics to continue a treatment focused on relapse prevention. Psychotropic medications and other psychosocial interventions were prescribed during the study. The first 4 (60-min) C-CT sessions took place biweekly and the last 6 sessions monthly. The visits of the patients of the fluoxetine and pill placebo groups occurred at the same frequency, the initial visit lasting 45 $\mathrm{min}$ and the sessions thereafter up to $30 \mathrm{~min}$. Fluoxetine and placebo were titrated upward; from week 8 , the modal dose was $40 \mathrm{mg} /$ day ( $73 \%$ of the patients reached this dose). At the end of 8 months, the treatments were stopped without a downward taper. Only 181 patients entered follow-up (70 in the C-CT, 62 in the fluoxetine, and 
49 in the pill placebo group), 145 completed at least 12 months of follow-up (56 in the C-CT, 52 in the fluoxetine, and 37 in the pill placebo group), and 124 completed 24 months (47 in the C-CT, 44 in the fluoxetine, and 33 in pill placebo group). As predicted, the C-CT and fluoxetine groups were significantly less likely to relapse than the pill placebo group. Across the 8 months of continuation treatment, the relapse/recurrence rates for $\mathrm{C}-\mathrm{CT}$ and fluoxetine were almost identical, though the C-CT patients stayed in treatment longer and attended more sessions than those in the other two groups. However, contrary to prediction, the relapse/recurrence rates following the discontinuation of C-CT and fluoxetine did not differ. It is important to note that 2 patients were hospitalized during acute-phase CT for worsening depression and/or suicidal ideation; they were withdrawn from the study and treated accordingly. During the continuation phase, 1 patient on placebo was hospitalized for suicidal ideation and withdrawn from the study. Three patients (1 from each group) were hospitalized during follow-up due to worsening depression and/or suicidal ideation. Though the generalizability of the results is limited (e.g., patients being mostly white females in their early 40 s, highly trained therapists not always available in the community), the results have important implications emphasized by the authors: (1) patients who respond to CT but remain at higher risk of relapse/recurrence not only benefit from C-CT but obtain comparable prophylaxis from fluoxetine; (2) continuation-phase fluoxetine alone can also be used to reduce the risk of relapse after an initial course of CT; (3) although fluoxetine was effective, patients in the pharmacotherapy arms were more likely to drop out during the first month of the experimental phase; (4) there was no evidence that C-CT conveyed more durable prophylaxis in acute-phase CT responders after treatment was stopped than fluoxetine; (5) the placebo data after the end of the experimental phase are difficult to interpret as placebo group patients relapsed early in that phase, and (6) even patients judged to be at high risk for relapse/recurrence after CT had a relatively low risk of relapse after continuation-phase therapies were stopped. (This study was supported by several grants from the NIMH and by unrestricted support from Eli Lilly and Company, which provided fluoxetine and matched pill placebo for the first 6 years of the study. Thereafter, the study materials were purchased and prepared to appear identical for both sites of the study by the pharmacy at the University of Texas Southwestern Medical Center.)

Beardslee et al. [25] presented the results of a continuation of their previous report [26] on the prevention of depression in at-risk adolescents. In the original study [26], 316 adolescent offspring (aged 13-17 years) of parents with current and/or prior depressive disorder were assigned either to a group cognitive-behavioral prevention (CBP) program (8 weekly 90 -min sessions followed by 6 monthly discontinuation sessions) or usual care (UC). The patients had histories of depression, current elevated depressive symptoms, or both but did not currently meet the criteria for a depressive disorder. The primary outcome was a probable or definite episode of depression (Depression Symptom Rating Scale score $>4$ ) for at least 2 weeks through a 9-month follow-up. At this 9-month follow-up, adolescents randomized to the CBP had significantly fewer episodes of depression (21.4\%) than those in UC (32.7\%). Baseline parental depression significantly moderated the effect: for adolescents whose parents were not depressed at intake, the CBP was significantly better in preventing subsequent episodes of depression than UC, while among adolescents whose parents were depressed at baseline, there was no difference in preventing reoccurrence between the CBP and UC. In the 33-month follow-up reported here [25], the results were pretty similar: there was no difference between the $\mathrm{CBP}$ and UC in adolescents whose parents were actively depressed at baseline, while the CBP was superior to UC in adolescents whose parents were not depressed at intake. (This study was supported by four NIMH grants and by a grant from the National Center for Research Resources.)

As Berking et al. [27] point out, there is evidence supporting the notion that deficits in emotion regulation (ER) help maintain major depressive disorder (MDD) and that modifying maladaptive ER strategies may lead to a reduction of depressive symptomatology. Thus, as they claim, systematically enhancing ER skills may improve the efficacy of CBT for depression. Therefore, they conducted a randomized controlled trial of 432 inpatients with MDD. Routine CBT patients (237 initially, 229 completed) received at least one 45 -min session of individual therapy and four 45-min sessions of group therapy (structured through modules used in CBT) as well as 4 sessions of transdiagnostic group therapy based on problem-solving therapy. Patients on routine CBT supplemented with ER skills training (ERT; 195, 186 of them completers) had parts of the CBT replaced by a systematic ERT which consisted of four 1.5-hour sessions and two 45-min sessions. When compared with routine CBT patients, those in the CBT-ERT group demonstrated a significantly greater reduction in depression ( 84.9 vs. $75.5 \%$ response rate; 65.1 vs. $51.1 \%$ remission rate), a greater reduction in negative 
affect, and a greater increase in well-being and ER skills. The results suggest that integrating strategies that target ER skills improves the efficacy of CBT in MDD. However interesting, this well-designed study with good retention rates and good size sample populations has severe limitations, such as using only a self-assessment instrument for treatment outcome evaluation. The main limitations to generalizability (the costs and inpatient location) are not mentioned. (The preparation of this paper was supported by the Vogelsberg Clinic and two Swiss National Science Foundation grants.)

CBT or CT is clearly efficacious in major depression and the prevention of relapse, recurrence, or occurrence of new symptoms (in adolescents). Its efficacy could be enhanced with the addition of ERT. However, CBT is not always better than other psychotherapy modalities or antidepressants. It is also noteworthy that CBT and other therapies could be associated with negative outcomes/adverse effects.

\section{Miscellaneous}

At the end, I would like to mention three rather thought-provoking articles, one of them a previously commented review published in this journal (though I usually do not use review articles) and another one an editorial in this journal (though I also usually do not use editorials). Butterworth et al. [28] analyzed data from the English Adult Psychiatric Morbidity Survey in regard to the prevalence of common mental disorders among 2,603 responders between the ages of 21 and 54 years who were either employed or unemployed and looking for work at the time of the interview in 2007. The prevalence of common mental disorders was similar among those who were employed and those in the poorest-quality jobs, and both of these groups were more likely to experience a common mental disorder than those who were in highquality work. As the authors note, although employment is thought to promote mental health and well-being, work of poor psychosocial quality is not associated with any better mental health than unemployment. Thus, strategies focusing on the mental health benefits of employment should take into account the fact that these benefits only apply to those jobs with a positive psychosocial environment. (The data are available from the Economic and Social Data Service, a collaboration between the Universities of Essex and Manchester. Two authors were supported by Australian National Health and Medical Research Council Fellowships.)
A systematic review and meta-analysis by Offidani et al. [29] pointed out a paucity of studies directly comparing antidepressants and benzodiazepines in anxiety disorders. The superiority of antidepressants over benzodiazepines in terms of their efficacy and tolerability has not been supported by the available evidence. Studies comparing newer antidepressants and benzodiazepines actually showed comparable or greater improvement and fewer adverse effects in patients suffering from generalized anxiety disorder or panic disorder treated with benzodiazepines. Benzodiazepines have frequently been described as medications associated with serious clinical problems. For instance, a lot has been written about the benzodiazepine discontinuation syndrome, but is it really more serious than the venlafaxine discontinuation syndrome? It is remarkable that a massive change in prescribing patterns favoring newer antidepressants (e.g., SSRIs) over benzodiazepines in the treatment of anxiety disorders has happened, yet no solid comparative studies have been conducted. Benzodiazepines are valuable parts of our armamentarium, and their reassessment and reappraisal is warranted. (This review was supported in part by a grant from the Fondazione Cassa di Risparmio di Cesena.)

Finally, Rafanelli et al. [30], in an editorial in this journal, questioned the massive use of antidepressants in the cardiology setting. They noted that antidepressants have a modest effect in improving depression and lack an effect on cardiovascular prognosis in coronary artery disease (CAD) patients. They also noted that psychotherapy failed to affect cardiac prognosis in these patients as well and suggest that we need to reassess the type A behavior (free-floating hostility, time urgency, and competitiveness) in cardiac patients. This behavior is associated with an increased risk of cardiovascular disease and overlaps with mania and hyperthymic temperament (both not the best targets for antidepressants). According to the authors, depression in CAD patients may be the final common pathway of a number of psychosomatic developments and type A behavior and, thus, a misleading target for treatment. Therefore, different treatments for psychological symptoms associated with CAD should be used. The question is: which ones? (This work was supported in part by a grant from the Compagnia di San Paolo.)

\section{Disclosure Statement}

The author declares no conflict of interest. 


\section{References}

1 Balon R: Clinical factor 2010. Psychother Psy- 13 Christensen J, Grønborg TK, Sørensen MJ, chosom 2011;80:195-198.

-2 Balon R: Clinical factor 2011. Psychother Psychosom 2012;81:199-205.

3 Balon R: Clinical factor 2012. Psychother Psychosom 2013;82:204-212.

4 Fava GA: The clinical factor. Psychother Psychosom 2011;80:1-3.

5 Franko DL, Keshaviah A, Eddy KT, Krishna M, Davis MC, Keel PK, Herzog DB: A longitudinal investigation of mortality in anorexia nervosa and bulimia nervosa. Am J Psychiatry 2013;170:917-925. Schendel D, Parner ET, Pedersen LH, Vestergaard M: Prenatal valproate exposure and risk of autism spectrum disorders and childhood autism. JAMA 2013;309:1696-1703.

14 Meador KJ, Loring DW: Risk of in utero exposure to valproate. JAMA 2013;309:17301731.

15 Reisch T, Steffen T, Habenstein A, Tschacher $\mathrm{W}$ : Change in suicide rates in Switzerland before and after firearm restriction resulting from the 2003 'Army XXI' reform. Am J Psychiatry 2013;170:977-984.

6 Crow S: Eating disorders and risk of death. Am J Psychiatry 2013;170:824-825.

7 Dalle Grave R, Calugi S, Conti M, Doll H, Fairburn CG: Inpatient cognitive behavior therapy for anorexia nervosa: a randomized controlled trial. Psychother Psychosom 2013; 82:390-398.

$>8$ Touyz S, Le Grange D, Lacey H, Hay P, Smith R, Maguire S, Bamford B, Pike KM, Crosby $\mathrm{RD}$ : Treating severe and enduring anorexia nervosa: a randomized controlled trial. Psychol Med 2013;43:2501-2511.

$\checkmark 9$ Pearson RM, Evans J, Kounali D, Lewis G, Heron J, Ramchandani PG, O'Connor TG, Stein A: Maternal depression during pregnancy and postnatal period: risks and possible mechanisms for offspring depression at age 18 years. JAMA Psychiatry 2013;70:13121319.

$>10$ Stephansson O, Kieler H, Haglund B, Artama M, Engeland A, Furu K, Gissler M, Norgaard M, Nielsen RB, Zoega G, Valdimarsdottir U: Selective serotonin reuptake inhibitors during pregnancy and risk of stillbirth and infant mortality. JAMA 2013;303:48-54.

$>11$ Wisner KL, Bogen DL, Sit D, McShea M, Hughes C, Rizzo D, Confer A, Luther J, Eng H, Wisniewski SW: Does fetal exposure to SSRIs or maternal depression impact infant growth? Am J Psychiatry 2013;170:485-493.

12 Parry BL: To treat or not to treat perinatal depression with antidepressant medication: effects on infant growth. Am J Psychiatry 2013; 170:453-454.
6 Mann JJ, Gibbons RD: Guns and suicide. Am J Psychiatry 2013;170:939-941.

7 Kelleher I, Corcoran P, Keeley H, Wigman JT, Devlin N, Ramsay H, Wasserman C, Varli V, Cannon M: Psychotic symptoms and population risk for suicide attempt: a prospective cohort study. JAMA Psychiatry 2013;70:940948

18 Roberts SE, Jaremin B, Lloyd K: High-risk occupation for suicide. Psychol Med 2013;43: 1231-1240.

19 Hasselbach BJ, Knorr U, Hasselbach SG, Gade A, Kessing LV: The cumulative load of depressive illness is associated with cognitive function in the remitted state of unipolar depressive disorder. Eur Psychiatry 2013;28: 349-355.

20 Mora E, Portella MJ, Forcada I, Vieta E, Mur M: Persistence of cognitive impairment and its negative impact on psychosocial functioning in lithium-treated, euthymic bipolar patients: a 6-year follow-up study. Psychol Med

21 Dregan A, Gulliford MC: Leisure-time physical activity over the life course and cognitive functioning in late mid-adult years: a cohortbased investigation. Psychol Med 2013;43: 2447-2458.

22 Driessen E, Van HL, Don FJ, Peen J, Kool S, Westra D, Hendriksen M, Schoevers RA, Cuijpers P, Twisk JWR, Dekker JJM: The efficacy of cognitive-behavioral therapy and psychodynamic therapy in the outpatient treatment of major depression: a randomized clinical trial. Am J Psychiatry 2013;170:10411050 . Sarchiapone M, Hoven C, Wasserman D, 2013;43:1187-1196.
23 Stangier U, Hilling C, Heidenreich T, Risch AK, Barocka A, Schlosser R, Kronfeld K, Ruckers C, Berger H, Roschke J, Weck F, Volk S, Hambrecht M: Maintenance cognitive-behavioral therapy and manualized psychoeducation in the treatment of recurrent depression: a multicenter prospective randomized controlled trial. Am J Psychiatry 2013;170: 624-632.

24 Jarrett RB, Minhajuddin A, Gershenfeld H, Friedman ES, Thase ME: Preventing depressive relapse and recurrence in high-risk cognitive therapy responders: a randomized trial of continuation phase cognitive therapy, fluoxetine, or matched pill placebo. JAMA Psychiatry 2013;70:1152-1160.

-25 Beardslee WR, Brent DA, Weersing VR, Clarke GN, Porta G, Hollon SD, Gladstone TRG, Gallop R, Lynch FL, Iyengar S, DeBar L, Garber J: Prevention of depression in at-risk adolescents: long-term effects. JAMA Psychiatry 2013;70:1161-1170.

26 Garber J, Clarke GN, Weersing VR, Beardslee WR, Brent DA, Gladstone TR, DeBar LL, Lynch FL, D’Angelo E, Hollon SD, Shamseddeen W, Iyengar S: Prevention of depression in at-risk adolescents: a randomized controlled trial. JAMA 2009;301:2215-2224.

27 Berking M, Ebert D, Cuijpers P, Hofmann S: Emotion regulation skills training enhances the efficacy of inpatient cognitive behavioral therapy for major depressive disorder: a randomized controlled trial. Psychother Psychosom 2013;82:234-245.

28 Butterworth P, Leach LS, McManus S, Stansfeld SA: Common mental disorders, unemployment and psychosocial job quality: is a poor job better than no job at all? Psychol Med 2013;43:1763-1772.

29 Offidani E, Guidi J, Tomba E, Fava GA: Efficacy and tolerability of benzodiazepines versus antidepressants in anxiety disorders: a systematic review and meta-analysis. Psychother Psychosom 2013;82:355-362.

30 Rafanelli C, Sirri L, Grandi S, Fava GA: Is depression the wrong treatment target for improving outcome in coronary artery disease? Psychother Psychosom 2013;82:285-291. 